\title{
Olfactory receptor 544 reduces adiposity by steering fuel preference toward fats
}

\author{
Chunyan Wu, ${ }^{1}$ Su Hyeon Hwang, ${ }^{1}$ Yaoyao Jia, ${ }^{1}$ Joobong Choi, ${ }^{1}$ Yeon-Ji Kim, ${ }^{1}$ Dahee Choi, ${ }^{2}$ Duleepa Pathiraja, In-Geol Choi, ${ }^{1}$ \\ Seung-Hoi Koo, ${ }^{2}$ and Sung-Joon Lee ${ }^{1}$
}

'Department of Biotechnology and 2Division of Life Sciences, School of Life Sciences and Biotechnology for BK21 PLUS, Korea University, Seoul, Republic of Korea.

\begin{abstract}
Olfactory receptors (ORs) are present in tissues outside the olfactory system; however, the function of these receptors remains relatively unknown. Here, we determined that olfactory receptor $\mathbf{5 4 4}$ (Olfr544) is highly expressed in the liver and adipose tissue of mice and regulates cellular energy metabolism and obesity. Azelaic acid (AzA), an Olfr544 ligand, specifically induced PKA-dependent lipolysis in adipocytes and promoted fatty acid oxidation (FAO) and ketogenesis in liver, thus shifting the fuel preference to fats. After 6 weeks of administration, mice fed a high-fat diet (HFD) exhibited a marked reduction in adiposity. AzA treatment induced expression of PPAR- $\alpha$ and genes required for FAO in the liver and induced the expression of PPAR- $\gamma$ coactivator 1- $\alpha$ (Ppargc1a) and uncoupling protein-1 (Ucp1) genes in brown adipose tissue (BAT). Moreover, treatment with AzA increased insulin sensitivity and ketone body levels. This led to a reduction in the respiratory quotient and an increase in the $\mathrm{FAO}$ rate, as indicated by indirect calorimetry. AzA treatment had similar antiobesogenic effects in HFD-fed ob/ob mice. Importantly, AzA-associated metabolic changes were completely abrogated in HFD-fed Olfr544 ${ }^{-/-}$mice. To our knowledge, this is the first report to show that Olfr544 orchestrates the metabolic interplay between the liver and adipose tissue, mobilizing stored fats from adipose tissue and shifting the fuel preference to fats in the liver and BAT.
\end{abstract}

\section{Introduction}

Olfactory receptors (ORs) are classic GPCRs primarily expressed on olfactory sensory neuron cilia located in the olfactory epithelium (1). OR genes constitute the largest gene family in mammals, and approximately 1,200 OR genes are present in mice and 400 in humans (2). Activation of ORs in the nose triggers intracellular signal transduction pathways that ultimately lead to the recognition of and distinction between diverse volatile odorants in the environment (3). However, ORs are also expressed in various nonolfactory tissues, in which their functions are not associated with chemosensation (4).

Recent studies have highlighted the finding that ORs have diverse functions in various tissues in connection to several physiological contexts beyond odor recognition: MOR17-4 controls cellular motility and chemotaxis in sperm cells (5); OR1A1 modulates hepatic triglyceride metabolism in human hepatocytes through the PKA signaling pathway (6); Olfr78 functions as a renal short-chain fatty acid receptor involved in the regulation of renin secretion and blood pressure in the renal juxtaglomerular apparatus (7) and as a hypoxia sensor in the breathing circuit and stimulator of carotid sinus nerve activity in response to lactate in glomus cells (8); and MOR23 mediates signal transduction in myocytes for muscle regeneration and repair in skeletal muscle (9). These reports suggest that the diverse functions

Conflict of interest: The authors have declared that no conflict of interest exists. Submitted: July 6, 2016; Accepted: August 29, 2017.

Reference information: / Clin Invest. 2017;127(11):4118-4123.

https://doi.org/10.1172/JCl89344. of ORs might include the regulation of energy metabolism; however, the roles of ORs in metabolic tissues are largely unknown. We sought to determine the biological functions of ectopically expressed ORs in liver and adipose tissue, the 2 major tissues in the regulation of energy metabolism.

\section{Results and Discussion}

Olfactory receptor 544 is functionally expressed in mouse liver and adipose tissue. To identify ORs highly expressed in the liver and adipose tissue, we initially conducted a microarray analysis of mice fed a normal diet (ND) of regular chow or a high-fat diet (HFD) (data not shown); the highly expressed ORs were confirmed by quantitative PCR (qPCR) analysis. Among ORs expressed in liver and adipose tissue, olfactory receptor 544 (Olfr544) showed the highest expression in the liver in both ND- and HFD-fed mice and in adipose tissue of HFD-fed mice according to qPCR analysis (Supplemental Figure 1, A and B; supplemental material available online with this article; https:// doi.org/10.1172/JCI89344DS1). Olfr544 was also expressed in the heart, brain, spleen, and colon (Supplemental Figure 1C). We also detected Olfr544 mRNA expression in primary hepatocytes, cultured Hepa1c1c-7 mouse hepatocytes, 3T3-L1 mouse adipocytes, and mouse interscapular brown adipose tissue (BAT) (Supplemental Figure 2, A and B). The OR signaling components $\mathrm{G}_{\text {olfo }}(\mathrm{Gnal})$ and adenylate cyclase-3 (Adcy3) were coexpressed in Hepa1c1c-7 and 3T3-L1 cells, suggesting that Olfr544 signaling functions in these cells (Supplemental Figure 2C). Collectively, these results indicate that Olfr544 is functionally expressed in both cell types. 
A
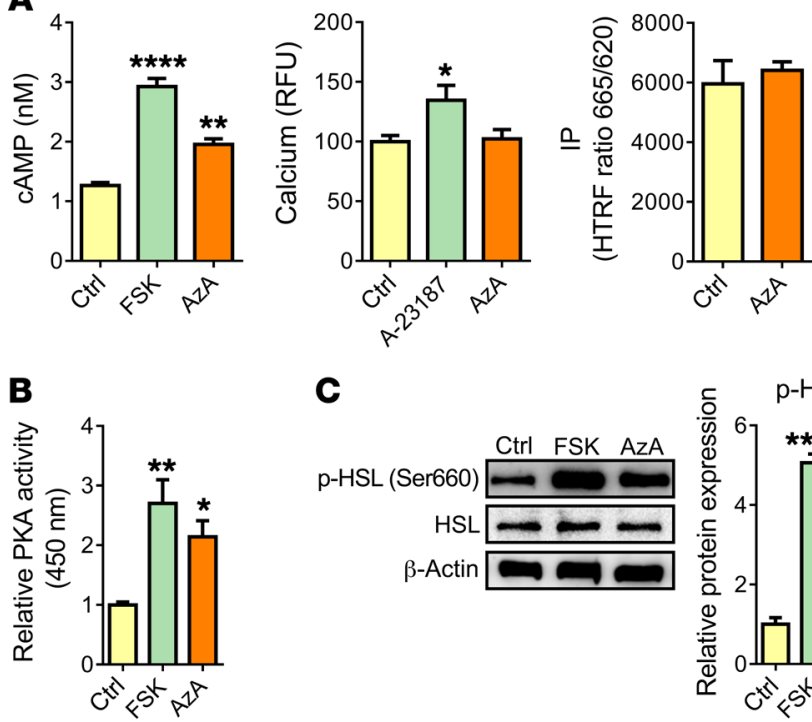

C
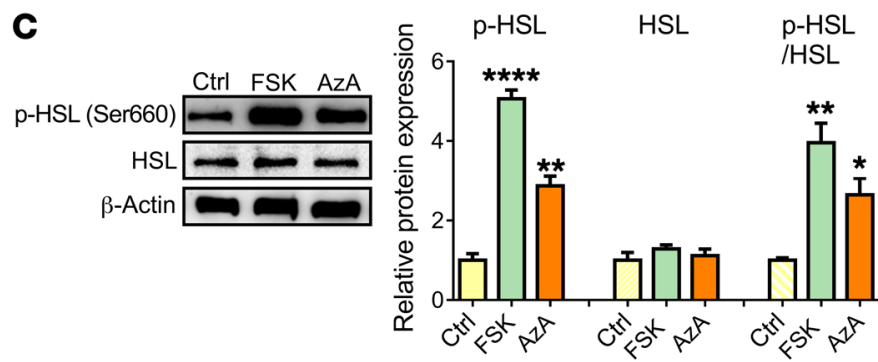

E
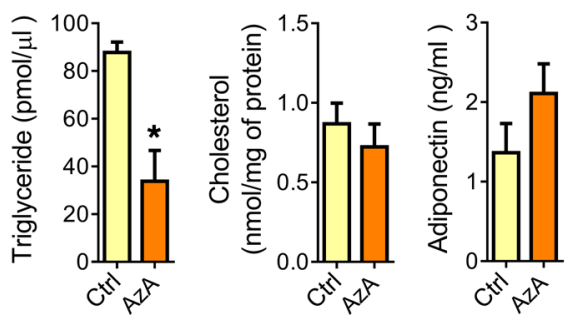

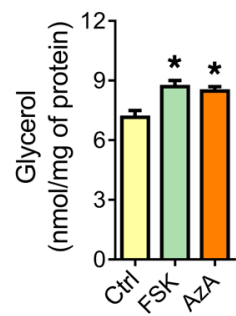

Figure 1. Olfr544 activation by AzA stimulates lipolysis in 3T3-L1 adipocytes. (A) AzA increases CAMP concentrations $(n=4)$, but not intracellular calcium $(n=8)$ or inositol phosphate (IP) levels $(n=4)$. HTRF ratio, homogeneous time-resolved fluorescence ratio $(665 \mathrm{~nm} / 620 \mathrm{~nm})$; RFU, relative fluorescence units. (B) PKA activity $(n=4)$. Relative PKA activity was measured using absorbance at $450 \mathrm{~nm}$ and normalized to the protein concentration. (C) Immunoblot analysis of phosphorylated HSL ( $p-H S L), H S L$, and the p-HSL/HSL ratio $(n=3)$. (D) Intracellular triglyceride $(n=3)$ and cholesterol $(n=4)$ concentrations and adiponectin secretion $(n=4)$. (E) Glycerol release $(n=4)$. FSK, forskolin (1 $\mu \mathrm{M}) ;$ AzA (50 $\mu \mathrm{M}) ;$ A-23187 $(10 \mu \mathrm{M})$, a calcium-ionophore positive control. Data are presented as the mean \pm SEM. ${ }^{*} P<0.05$ ${ }^{* *} P<0.01$, and ${ }^{* * *} P<0.0001$, by 1 -way ANOVA followed by Tukey's HSD test or Student's $t$ test for comparison of 2 or more groups.
Activation of Olfr544 by azelaic acid stimulates cAMP response element-binding protein activity. OR activation stimulated by ligands triggers multiple signaling pathways. Azelaic acid (AzA) and suberic acid are known Olfr544 ligands $(10,11)$. We examined the effect of Olfr544 in Hana3A cells transfected with N-terminal Lucy-FLAG-tagged Olfr544. Hana3A cells and the N-terminal Lucy-FLAG tags are known to improve the heterologous expression of ORs $(12,13)$, and we found that the expression levels of Olfr544 and Olfr545 mRNA and proteins in cell membrane were similar (Supplemental Figure 3, $A$ and $B)$. AzA and suberic acid induced cAMP response element-binding protein (CREB) activity (the concentration of a compound where $50 \%$ of its maximal effect $\left[\mathrm{EC}_{50}\right]=29.7$ and $537.4 \mu \mathrm{M}$, respectively) in Olfr544-overexpressing cells, but not in cells overexpressing Olfr545, which has high homology with Olfr544 (Supplemental Figure 3C). The AzA-induced CREB activity was significantly blunted in cells cotreated with the adenylate cyclase inhibitor SQ22536 (Supplemental Figure 3, D and E). These indicated that AzA activates the Olfr544/ cAMP/PKA/CREB signaling axis. AzA increased cAMP levels but not those of calcium or inositol phosphates (Supplemental Figure 3F), and similar effects of AzA were observed in cultured Hepa1c1c-7 hepatocytes. AzA increased cAMP levels but not intracellular calcium or inositol phosphate concentrations and stimulated CREB phosphorylation, all of which were negated with Olfr544 gene knockdown (Supplemental Figure 4). Collectively, these results indicate that AzA-activated Olfr544 stimulates CREB activity.
Olfr544 stimulates the PKA-hormone-sensitive lipase signaling axis and lipolysis. Next, we investigated the biological functions of Olfr544 and AzA in 3T3-L1 adipocytes. AzA increased the cAMP concentration, but not that of calcium or inositol phosphates (Figure 1A), thus activating PKA and increasing phosphorylation of hormone-sensitive lipase (HSL) at serine 660 in 3T3-L1 adipocytes (Figure 1, B and C). Interestingly, AzA lowered the intracellular triglyceride concentration, with marginal changes in cholesterol levels and adiponectin secretion (Figure 1D). This hypolipidemic effect of AzA was mediated by increased lipolysis, as indicated by an increase in glycerol release (Figure 1E). Olfr544 mRNA expression was reduced by $80 \%$ after knockdown in 3T3-L1 adipocytes (Supplemental Figure 5A). Under these conditions, intracellular cAMP concentration, PKA activity, HSL phosphorylation, and glycerol release were not altered by AzA in 3T3-L1 cells (Supplemental Figure 5, B-D). These findings demonstrate that AzA-activated Olfr544 stimulates the PKA/HSL signaling axis and induces lipolysis in cultured adipocytes. We examined the acute metabolic functions of Olfr544 and AzA in C57BL/6J WT and Olfr544-/mice. We generated Olfr $544^{-/-}$mice by deleting base pairs (bp) 161-428 of exon 2 using the CRISPR/Cas9 system (Supplemental Figure 6 and Supplemental Figure 7A); deletion of exon 2 in the Olfr544 gene was confirmed by PCR, DNA sequencing analyses, and off-target sequencing (Supplemental Figure 7, B-D). The appearance, birth rate, and growth of Olfr $544^{-/-}$mice were normal. Acute AzA injection induced lipolysis in WT mice, but this effect was abrogated in Olfr $544^{-/-}$mice (Figure 2A). 
A

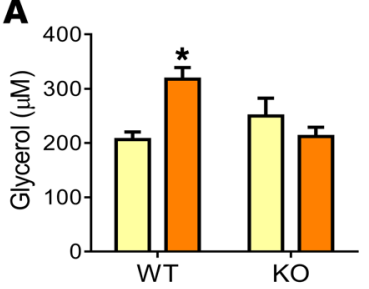

B
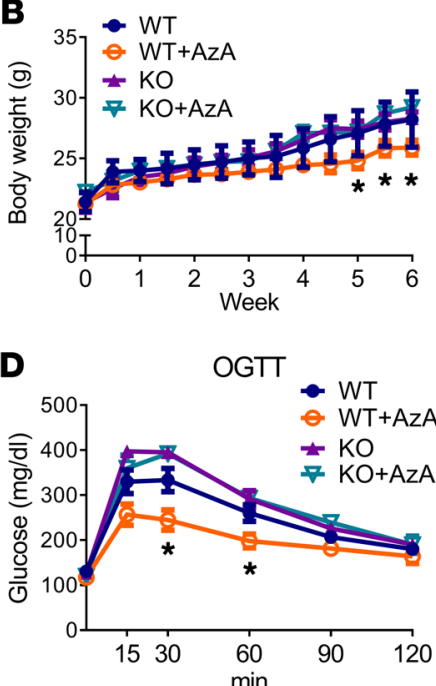

C
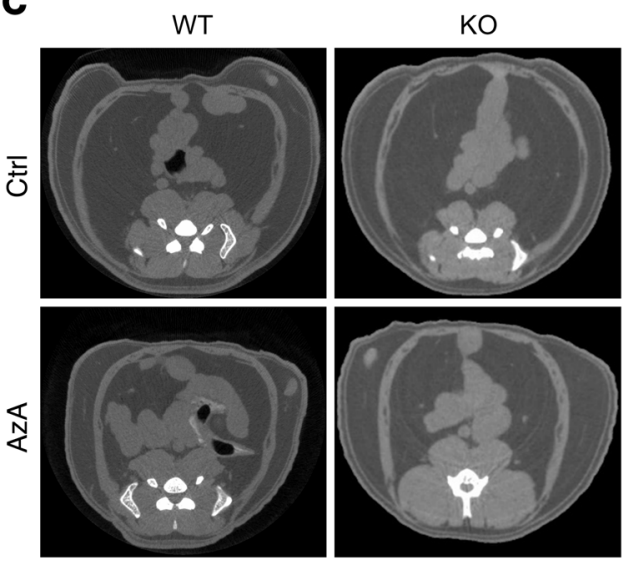
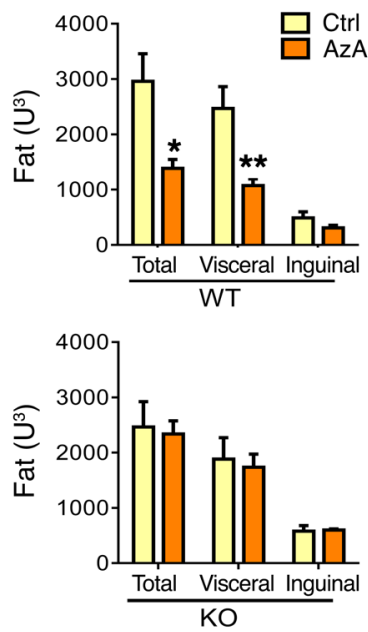

E
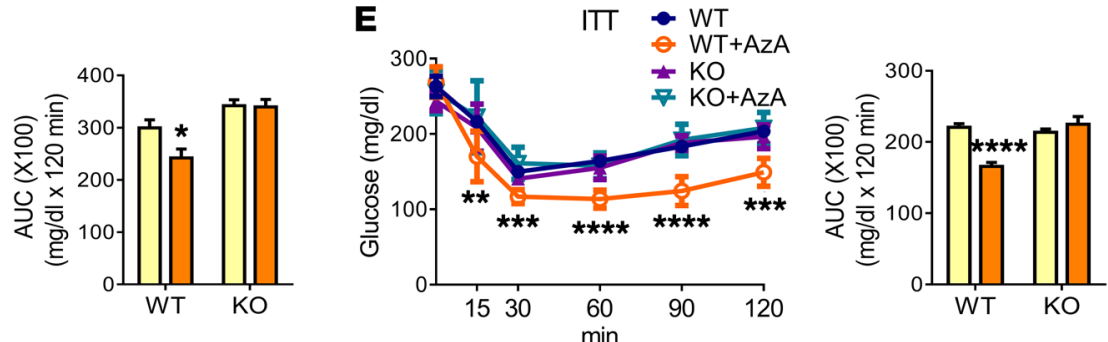

Figure 2. AzA administration reduces adiposity and improves insulin sensitivity in HFD-fed WT mice but not HFD-fed OIfr544-/- mice. (A) Lipolysis in WT and Olfr544-/- $($ KO) mice $(n=4)$. (B) Body weight and (C) adipocyte tissue mass assessed by micro-CT in HFD-fed WT ( $n=7-8)$ and Olfr544/- $(n=4-5)$ mice after 6 weeks of treatment with AzA (50 mg/kg of body weight/day). Adipose tissue appears dark gray. (D) Oral glucose tolerance test (OCTT) in HFD-fed mice at 5 weeks after overnight fasting and (E) insulin tolerance test (ITT) in HFD-fed mice at 5.5 weeks after a 4-hour fast ( $n=7-8$ for WT mice and $n=5$ for Olfr544 ${ }^{-/-}$mice). Data are presented as the mean \pm SEM. The HFD used in this study was a rodent diet containing $60 \%$ of calories from fat. ${ }^{*} P<0.05$, ${ }^{* *} P<0.01$, ${ }^{* *} P<0.001$, and ${ }^{* * * *} P<0.0001$, by Student's $t$ test or 1 -way ANOVA followed by Tukey's HSD test for comparison of 2 or more groups.

No difference in glycerol levels was detected between the WT and Olfr $544^{-/-}$control groups. These findings indicate that AzA induces the Olfr544/PKA/HSL signaling axis and increases adipose lipolysis as an acute effect in vivo.

Long-term AzA-induced activation of Olfr544 reduces adiposity, increases fatty acid oxidation, and improves glucose and insulin tolerance in HFD-fed mice. We studied the long-term effects of Olfr544 activation by orally administering AzA to HFD-fed WT and Olfr $544^{-/-}$mice for 6 weeks. AzA (50 mg/kg body weight) significantly reduced the body weight and visceral adiposity of HFDfed WT mice, but not of HFD-fed Olfr $544^{-/-}$mice, demonstrating the antiobesogenic effects of AzA (Figure 2, B and C), without affecting food intake (Supplemental Table 1). The size of visceral and inguinal adipocytes was also reduced in HFD-fed WT mice (Supplemental Figure 8A). Administration of AzA significantly improved glucose and insulin tolerance in HFD-fed WT mice (Figure 2, D and E), as plasma glucose and insulin levels decreased and the insulin resistance index improved (Supplemental Figure 8B). We found that plasma triglyceride, cholesterol, adiponectin, glucagon-like peptide-1, and glycogen levels were unaltered (data not shown), whereas hepatic triglyceride concentrations decreased and ketone body concentrations increased in HFD-fed WT mice administered AzA (Supplemental Figure 8C). However, these metabolic changes caused by AzA disappeared in HFD-fed Olfr544-/- mice (Figure 2, B-E and Supplemental Figure 8). AzA showed similar antiobesogenic effects in HFD-fed $o b / o b$ mice, without significantly altering food intake (Supplemental Figure 9 and Supplemental Table 1). Collectively, these results demonstrate that long-term activation of Olfr544 by AzA has antiobesogenic effects by inducing adipose tissue lipolysis and ketogenesis. These metabolic alterations improved glucose tolerance.

AzA induces hepatic PPAR- $\alpha$ expression, which increases hepatic fatty acid oxidation and brown adipose thermogenesis in HFD-fed mice. Next, we investigated the potential antiobesogenic mechanism of Olfr544 activation in HFD-fed WT mice. The results of a gene expression analysis showed that AzA significantly induced PPAR- $\gamma$ coactivator 1- $\alpha$ (Ppargc1a) and uncoupling protein-1 (Ucp1) expression in interscapular BAT, but did not alter the expression of PR domain-containing 16 (Prdm16) or Ucp1 in inguinal adipocytes (Figure 3A). PPAR- $\alpha$ and its response genes were also induced in Hepa1c1c-7 hepatocytes and the livers of HFD-fed WT and $o b / o b$ mice by AzA administration (Figure 3B and Supplemental Figure 10A). PPAR- $\alpha$ is an essential regulator of $\beta$-oxidation; thus, we analyzed fatty acid oxidation (FAO) in the mouse liver. The results revealed that AzA administration significantly increased hepatic FAO in Hepa1c1c-7 cells and 

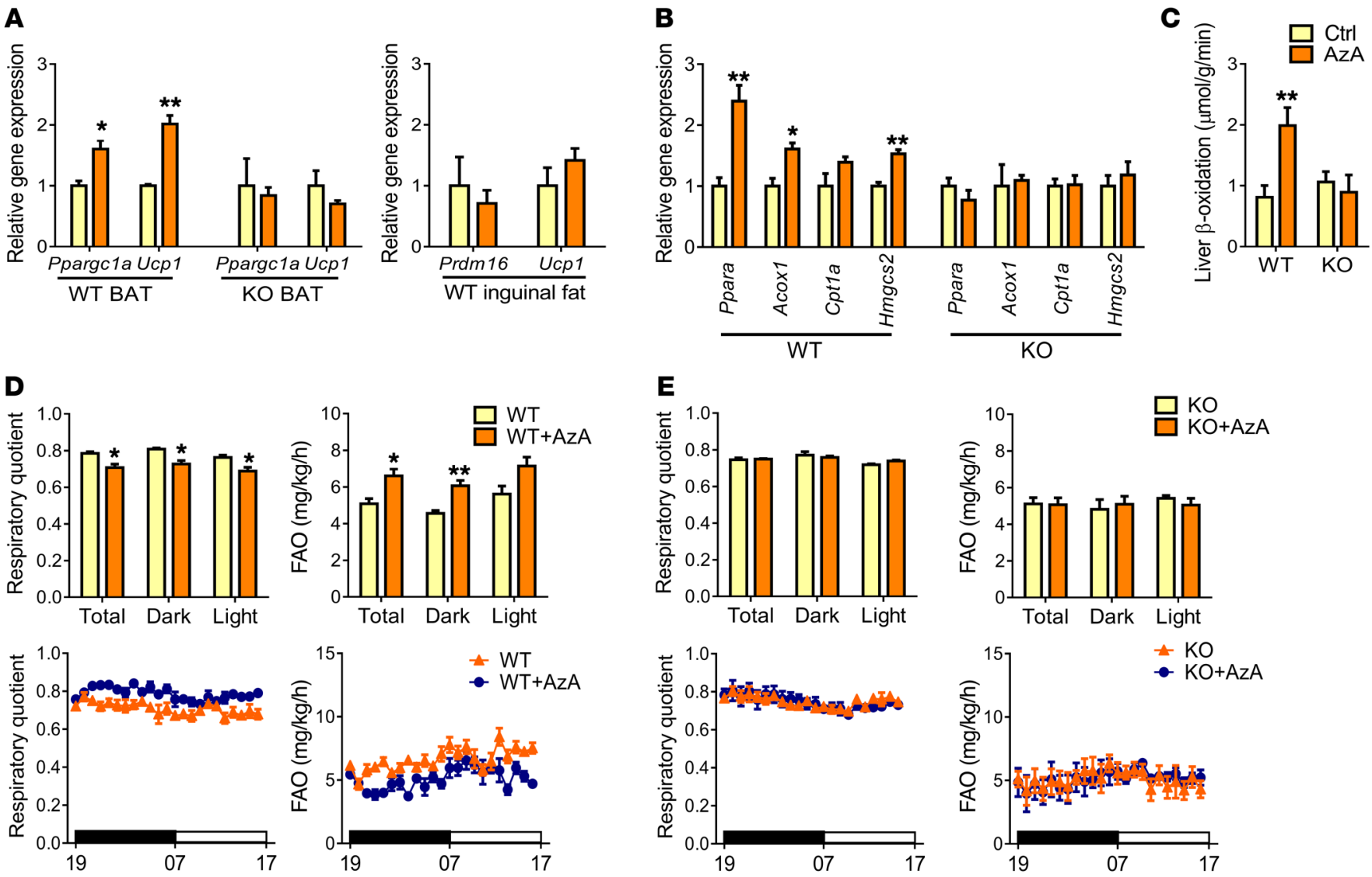

Figure 3. AzA administration induces hepatic PPAR- $\alpha$ expression and FAO and shifts the fuel preference to fats in HFD-fed WT mice but not in HFD-fed Olfr544-/- mice. (A) Expression levels of Ppargc1a and Ucp1 in HFD-fed mouse interscapular BAT and Prdm16 and Ucp1 expression in HFD-fed mouse inguinal adipose tissue $(n=3)$. (B) Expression levels of PPAR- $\alpha$ and its responsive genes $(n=3)$ in HFD-fed mouse livers. (C) FAO in HFD-fed WT ( $n=7-8)$ and Olfr544-/- $(n=5)$ mouse livers. (D) Indirect calorimetric analysis of HFD-fed WT and (E) Olfr544 ${ }^{-1-}(n=3-4)$ mice. Data are presented as the mean \pm SEM. ${ }^{*} P<0.05$ and ${ }^{* *} P<0.01$, by Student's $t$ test.

HFD-fed WT mouse livers (Figure 3C and Supplemental Figure 10B). In the luciferase assay, we found that AzA did not affect the transactivation of PPAR- $\alpha$ in cells transfected with both PPRE-luc and PPAR- $\alpha$ expression vectors, demonstrating that AzA is not an agonist for PPAR- $\alpha$ (Supplemental Figure 10C). In addition, indirect calorimetry analysis revealed that the respiratory quotient in the dark cycle was decreased significantly and that FAO was increased significantly (Figure 3D), without altering total energy expenditure or food intake (data not shown) in HFD-fed WT mice. However, these effects were completely abrogated in HFD-fed Olfr $544^{-/}$mice (Figure 3, A-C, and E). These results demonstrate that the antiobesogenic effects of AzA are primarily due to induced FAO and BAT thermogenesis, which occurs via Olfr544-activated PPAR- $\alpha$ induction.

AzA is a C9 dicarboxylic acid present in various grain foods including wheat, barley, and rye (14). Thus, it is possible that dietary AzA activates Olfr544. An in vitro cell viability test revealed no effect of AzA on Hepa1c1c-7 or 3T3-L1 cell viability, and liver enzyme levels in HFD-fed mice were unaffected by AzA (Supplemental Figure 11). We administered $50 \mathrm{mg} / \mathrm{kg}$ body weight AzA in the mouse studies. We chose this dose while taking into account the safety and efficacy of AzA in mice. Four previous mouse feeding studies used varying doses of AzA (20-417 mg/kg/mouse) with significant biological effects but no apparent toxicity (15-18). Although there has been no pharmacokinetic study of AzA and its bioavailability is unknown, the dose we selected ensures the $\mathrm{EC}_{50}$ value in vivo, even if its bioavailability is assumed to be $1 \%$. AzA can also be produced endogenously by hepatic $\omega$-oxidation, which is stimulated under ketogenic conditions of starvation and diabetes (19) and may represent a fasting and ketogenic signal to promote FAO. AzA has been detected in humans (20), suggesting that it could be an important metabolic regulator in the fasting and ketogenic state and may be safe for use. This possibility will be further investigated in the future.

Acute activation of Olfr544 by AzA specifically increased cAMP levels and stimulated PKA/HSL signaling in adipocytes, which induced lipolysis in vitro and in vivo. Long-term oral administration of AzA reduced adiposity in HFD-fed WT and $o b / o b$ mice and dramatically induced PPAR- $\alpha$ expression; thus, glycerol and free fatty acids excreted from adipose tissues can be consumed as fuel in the liver for FAO and ketogenesis. It has been reported that PKA activates PPAR- $\alpha$ by phosphorylation at serine 78 (21). In addition, PPAR- $\alpha$ gene expression requires transcription factors, including PPAR- $\alpha$ itself, CREB, CBP/P300, and CITED2 (a CBP/P300-interacting coactivator) (22). Therefore, PPAR- $\alpha$ gene expression can be stimulated by the Olfr544/ PKA/CREB signaling pathway, although the mechanism is not 
clearly understood. The induction of PPAR- $\alpha$ is of particular interest, because hepatic PPAR- $\alpha$ activates fatty acid uptake, FAO, and ketogenesis in the liver (23); thus, administration of fibrates as PPAR- $\alpha$ agonists reduces the accumulation of body fat (24). In addition, PPAR- $\alpha$ induces PDK4 expression, which favors fat consumption for energy production by inhibiting the pyruvate dehydrogenase complex through phosphorylation (25). Thus, activated hepatic Olfr544 preferentially oxidizes fatty acids mobilized from adipose tissues and reduces the adiposity of HFD-fed WT and $o b / o b$ mice by activating PPAR- $\alpha$ expression.

In the indirect calorimetric analysis, AzA reduced the respiratory quotient and increased $\mathrm{FAO}$, indicating that AzA promotes oxidation of fat rather than carbohydrate for energy production in HFD-fed mice. The induction of lipolysis and subsequent mobilization of lipids from adipose tissues to the liver for use as fuel, followed by preferential oxidation of fatty acids after PPAR- $\alpha$ is induced in the liver, is a potential body fat reduction mechanism. Moreover, we observed that the expression of key thermogenic genes was markedly induced in BAT, indicating that AzA reduces adiposity by increasing thermogenesis in BAT.

The insulin sensitivity index and glucose tolerance improved in HFD-fed WT, but not $o b / o b$, mice after the administration of AzA, which agrees with the findings of a previous study (17). These appear to be epiphenomenal results caused by a relatively large reduction of adiposity in WT mice. One study suggested the ectopic expression of Olfr544 in the $\alpha$ TC1-9 pancreatic $\alpha$-cell line. The study showed that AzA increases the intracellular calcium level so that it has a marginal role in glucagon secretion (11). These results suggest that activation of Olfr544 by AzA may be tissue specific.

In this study, we report that Olfr544, a GPCR thus far classified as an OR, is highly expressed in nonolfactory liver and adipose tissues and has functional relevance in energy metabolism and obesity. This is the first report to our knowledge of an ectopic OR playing a critical role in energy and lipid metabolism. We postulate that Olfr544 stimulates hepatic FAO of fuel molecules mobilized from adipose tissue by inducing lipolysis and thermogenesis in BAT, thus orchestrating metabolic coop- eration between the liver and adipose tissues, which contributes to reduce adiposity. Our findings suggest that Olfr544 may be a therapeutic target for treating obesity.

\section{Methods}

Further information can be found in the Supplemental Methods and in Supplemental Figures 1-11.

Accession number. Microarray data were deposited in the NCBI's Gene Expression Omnibus (GEO) database (GEO GSE102977).

Statistics. All data are shown as the mean \pm SEM, and a 2-tailed Student's $t$ test or 1-way ANOVA followed by Tukey's honest significant difference (HSD) test was used to compare 2 or more groups. A $P$ value of less than 0.05 was considered statistically significant.

Study approval. All animal experiments were conducted according to protocols approved by the Animal Experiment Committee of Korea University (protocol KUIACUC-20090420-4).

\section{Author contributions}

SJL, IGC, and SHK designed and supervised the project. CW, SHH, YJ, JC, YJK, DP, and DC performed experiments and acquired the data. SJL, CW, and SHK analyzed and interpreted the results. CW and SJL wrote the manuscript.

\section{Acknowledgments}

We thank Hiroaki Matsunami (Duke University Medical Center, Durham, NC, USA) for providing Hana3A cells; Jennifer Pluznick (Johns Hopkins University School of Medicine, Baltimore, MD, USA) for the Lucy-FLAG-tagged ORexpression vector; and Boram Mok and Ji-Hae Lee (Korea University, Seoul, South Korea) for technical assistance. This study was supported by a grant from the National Research Foundation (NRF) of Korea (NRF-2016R1A2A2A05005483) funded by the South Korean government (MSIP).

Address correspondence to: Sung-Joon Lee, Department of Biotechnology, School of Life Sciences and Biotechnology for BK21 PLUS, Korea University, Seoul 02841, Republic of Korea. Phone: 82.2.3290.3029; Email:junelee@korea.ac.kr.
1. Su CY, Menuz K, Carlson JR. Olfactory perception: receptors, cells, and circuits. Cell. 2009;139(1):45-59.

2. Buck LB. A novel multigene family may encode odorant receptors. Soc Gen Physiol Ser. 1992;47:39-51.

3. Firestein S. How the olfactory system makes sense of scents. Nature. 2001;413(6852):211-218.

4. Flegel C, Manteniotis S, Osthold S, Hatt H, Gisselmann G. Expression profile of ectopic olfactory receptors determined by deep sequencing. PLoS One. 2013;8(2):e55368.

5. Spehr M, et al. Identification of a testicular odorant receptor mediating human sperm chemotaxis. Science. 2003;299(5615):2054-2058.

6. Wu C, et al. Activation of OR1A1 suppresses PPAR- $\gamma$ expression by inducing HES- 1 in cultured hepatocytes. Int J Biochem Cell Biol. 2015;64:75-80.

7. Pluznick JL, et al. Olfactory receptor responding to gut microbiota-derived signals plays a role in renin secretion and blood pressure regulation.
Proc Natl Acad Sci USA. 2013;110(11):4410-4415.

8. Chag AJ, Ortega FE, Riegler J, Madison DV, Krasnow MA. Oxygen regulation of breathing through an olfactory receptor activated by lactate. Nature. 2015;527(7577):240-244.

9. Griffin CA, Kafadar KA, Pavlath GK. MOR23 promotes muscle regeneration and regulates cell adhesion and migration. Dev Cell. 2009;17(5):649-661.

10. Abaffy T, Malhotra A, Luetje CW. The molecular basis for ligand specificity in a mouse olfactory receptor: a network of functionally important residues. J Biol Chem. 2007;282(2):1216-1224.

11. Kang N, et al. Olfactory receptor Olfr544 responding to azelaic acid regulates glucagon secretion in $\alpha$-cells of mouse pancreatic islets. Biochem Biophys Res Commun. 2015;460(3):616-621.

12. Saito H, Kubota M, Roberts RW, Chi Q, Matsunami H. RTP family members induce functional expression of mammalian odorant receptors. Cell. 2004;119(5):679-691.

13. Shepard BD, Natarajan N, Protzko RJ, Acres
OW, Pluznick JL. A cleavable N-terminal signal peptide promotes widespread olfactory receptor surface expression in HEK293T cells. PLoS One. 2013;8(7):e68758.

14. Gallagher RS, Ananth R, Granger K, Bradley B, Anderson JV, Fuerst EP. Phenolic and shortchained aliphatic organic acid constituents of wild oat (Avena fatua L.) seeds. J Agric Food Chem. 2010;58(1):218-225.

15. Litvinov D, Selvarajan K, Garelnabi M, Brophy L, Parthasarathy S. Anti-atherosclerotic actions of azelaic acid, an end product of linoleic acid peroxidation, in mice. Atherosclerosis. 2010;209(2):449-454.

16. Muthulakshmi S, Saravanan R. Protective effects of azelaic acid against high-fat diet-induced oxidative stress in liver, kidney and heart of C57BL/6J mice. Mol Cell Biochem. 2013;377(1-2):23-33.

17. Muthulakshmi S, Saravanan R. Efficacy of azelaic acid on hepatic key enzymes of carbohydrate metabolism in high fat diet induced type 2 diabetic mice. Biochimie. 2013;95(6):1239-1244. 
18. Muthulakshmi S, Chakrabarti AK, Mukherjee S. Gene expression profile of high-fat diet-fed C57BL/6J mice: in search of potential role of azelaic acid. J Physiol Biochem. 2015;71(1):29-42.

19. Miura Y. The biological significance of $\omega$-oxidation of fatty acids. Proc Jpn Acad, Ser B, Phys Biol Sci. 2013;89(8):370-382.

20. Bondia-Pons I, et al. UPLC-QTOF/MS metabolic profiling unveils urinary changes in humans after a whole grain rye versus refined wheat bread inter- vention. Mol Nutr Food Res. 2013;57(3):412-422. 21. Burns KA, Vanden Heuvel JP. Modulation of PPAR activity via phosphorylation. Biochim Biophys Acta. 2007;1771(8):952-960.

22. Tien ES, Davis JW, Vanden Heuvel JP. Identification of the CREB-binding protein/p300-interacting protein CITED2 as a peroxisome proliferatoractivated receptor alpha coregulator. JBiol Chem. 2004;279(23):24053-24063.

23. Kersten S. Peroxisome proliferator activat- ed receptors and obesity. Eur J Pharmacol. 2002;440(2-3):223-234.

24. Kersten S, Desvergne B, Wahli W. Roles of PPARs in health and disease. Nature. 2000;405(6785):421-424.

25. Huang B, Wu P, Bowker-Kinley MM, Harris RA. Regulation of pyruvate dehydrogenase kinase expression by peroxisome proliferator-activated receptor-alpha ligands, glucocorticoids, and insulin. Diabetes. 2002;51(2):276-283. 